\title{
STRONG INFLUENCE OF Tb CONCENTRATION ON DOMAIN STRUCTURE AND KERR EFFECT OF Tb $\mathrm{T}_{\mathbf{x}} \mathrm{Fe}$ 1-x THIN FILMS
}

\author{
V. FLORESCU, C. RAU AND N. J. ZHENG \\ Rice University, Department of Physics and Rice Quantum Institute, \\ Houston, Texas $77251-1892$.
}

\begin{abstract}
Amorphous $\mathrm{TbFe}$ films with perpendicular magnetic anisotropy are attracting wide attention as promising candidates for erasable high - density magneto - optical recording and thermomagnetic printing. $\mathrm{Tb}_{\mathrm{x}} \mathrm{Fe}_{1-\mathrm{x}}$ thin films with $0 \leq \mathrm{x} \leq 0.37$ are deposited on glass substrates using r.f. sputtering. The magnetic remanent domain structures are studied at room temperature using the longitudinal and polar Kerr effect. Samples with $0 \leq x \leq 0.23$ exhibit magnetic in-plane anisotropy, while samples with $0.26 \leq x \leq 0.37$ possess uniaxial anisotropy with easy axis perpendicular to the film plane. The remanent domain structures depend strongly on Tb content. High remanence and high coercivity are found for samples with $0.27 \leq x \leq 0.34$ which is confirmed by additional vibrating sample magnetometer (VSM) measurements.
\end{abstract}

\section{INTRODUCTION}

Amorphous rare-earth-transition metal alloys are widely investigated for the use as magneto -optical recording media. ${ }^{1,2}$ Moreover it has been suggested that TbFe films could provide suitable media for magnetic printing. ${ }^{3,4}$ In magnetic printing, latent images are produced thermomagnetically and developed using magnetic toner particles. 5 For such an application, films with high remanence and/or sufficient thickness are required in order to create a strong force on the toner particles. The domain structure and magnetic properties (anisotropy energy) depend strongly on the composition ${ }^{6}$ and on preparation parameters (substrate temperature). ${ }^{7}$ In this paper, we report the influence of $\mathrm{Tb}$ concentration on the remanent domain structure and on the magnetic properties (saturation magnetization, coercivity and remanence) of thin TbFe films.

\section{EXPERIMENTAL}

In our experiments, we use $\mathrm{Tb}_{x} \mathrm{Fe}_{1-\mathrm{x}}$ thin films with a Tb content $\mathrm{x}$ between 0 and 0.37 . The deposition was performed by co-sputtering in a pure argon atmosphere ( $99.997 \%$ ) using a target-substrate (glass) distance of $35 \mathrm{~mm}$. These deposition parameters are used for all samples. Details about the sputtering conditions are reported elsewhere. ${ }^{8,9}$ The composition of the films is determined by using X-ray fluorescence analysis (XFA) where values of $x=0$, $0.17,0.23,0.26,0.27,0.30,0.31,0.34,0.37$ are found. Film thicknesses, $t$, are about $150 \mathrm{~nm}$. All the samples are coated with a thin $\mathrm{Al}_{2} \mathrm{O}_{3}$ protective layer. The size of the sample is $30 \times 30$ $\mathrm{mm}^{2}$. For further measurements, the samples are cut to a size $15 \times 15 \mathrm{~mm}^{2}$. Each set of 
samples is used for different measurements in order to obtain reliable data.. Auger electron spectroscopy (AES) measurements are performed in order to check composition data obtained by using XFA and to obtain data about films homogeneity in the growth direction. Using a DRON - 2 diffractometer, the angular distribution of $X$ - ray diffracted intensities is measured using $\mathrm{Mo} \mathrm{K} \alpha$ radiation up to :

$$
\mathrm{K}_{\max }=4 \pi \sin \theta_{\max } / \lambda=15 \AA
$$

The radiation is monochromatized by using a concave quartz crystal placed in front of a scintillation counter. Computerized data processing allowed for corrections of $X$-ray scattering in air and in the glass substrate as well as absorption in the film. The first two contributions are estimated by measuring, under the same experimental conditions an annealed $\mathrm{Au}$ foil and a clean substrate respectively. The absorption $\mu \cdot t$ in the film $(\mu=$ linear absorption coefficient $)$ is measured with Mo $\mathrm{K} \alpha$ radiation in transmission geometry with reference to a clean substrate. The remanent magnetic domain structure is directly observed by using the Kerr effect and a digital contrast enhancement scheme. ${ }^{10}$ The local hysteresis loops are studied by using a He-Ne laser in the longitudinal and polar Kerr - effect configuration. Additional magnetization $(\mathrm{M})$ versus magnetic field $(\mathrm{H})$ loop measurements were made with a vibrating sample magnetometer (VSM), calibrated with a Ni standard in a $20 \mathrm{kOe}$ applied field. All the samples are measured in two position, perpendicular and parallel to applied field.

\section{RESULTS AND DISCUSSION}

AES depth profile analyses shows that our films are homogeneous in the growth direction. The Fe and Tb concentration does not change with increasing film depth and the values are similar to those determined by XFA. X - ray diffractograms show that films with $0.17 \leq \mathrm{x} \leq$ 0.37 are perfectly amorphous. The diffractogram patterns are quite similar to those reported in ref 9 for TbFe amorphous alloys, showing a main peak at $\mathrm{K}=1.43 \AA$ and further weaker details. For films with $x$ ranging between 0.17 and 0.27 , the ratio $\mathrm{K}_{2} / \mathrm{K}_{1}$ is around 1.56 . For films with $x=0$, the $X$ - ray diffraction patterns indicates, as expected the presence of polycrystalline Fe. Fig. 1 shows, for $x=0$ four different remanent domain structures obtained with MOKE. Using longitudinal Kerr effect conditions various domain pattern are observed after reducing an applied magnetic field from saturation value to zero and by selecting four different in-plane directions.(see Figs. 1A-1D).. The single domain state at saturation breaks up into a multidomain configuration after demagnetizing the films by a reducing the applied magnetic field to zero. No perpendicular magnetization could be observed. However, there is hard to establish an orientation of the spontaneous magnetization of the domains versus crystallographic directions because of polycrystalline Fe sample. The domain structures presented in Fig. 1 are very close to those reported in ref.11( domains size of $2000 \AA$ thick,for a 81 - Permalloy film). We notice that for Permalloy film demagnetized in a $\mathrm{c}$ magnetic field along the easy axis, the obtained patterns are similar to those presented in our Fig IA. How ever, Permalloy films demagnetized in a $\mathrm{c}$ magnetic field along the hard axis, the obtained patterns are near identical to those shown in our Fig. 1C. This comparison suggests for our polycrystalline Fe sample a sort of hierarchy for the in - plane easy directions : not all are really easy directions. Fig. 2 shows the characteristic remanent domain structure for samples with $x$ $=0.23$ (A), 0.26 (B ), 0.34 (C) and 0.37 (D). The patterns from Fig. 2A are obtained by using longitudinal Kerr effect, while Fig.2 B-2 D, by using polar Kerr effect The type of remanent domain structure in Fig. 2 B-2 D are very different from the domains in Fig. 2A and Fig. 1 . It is clear that we have now a structure with free magnetic poles, that can be led into a magnetic 

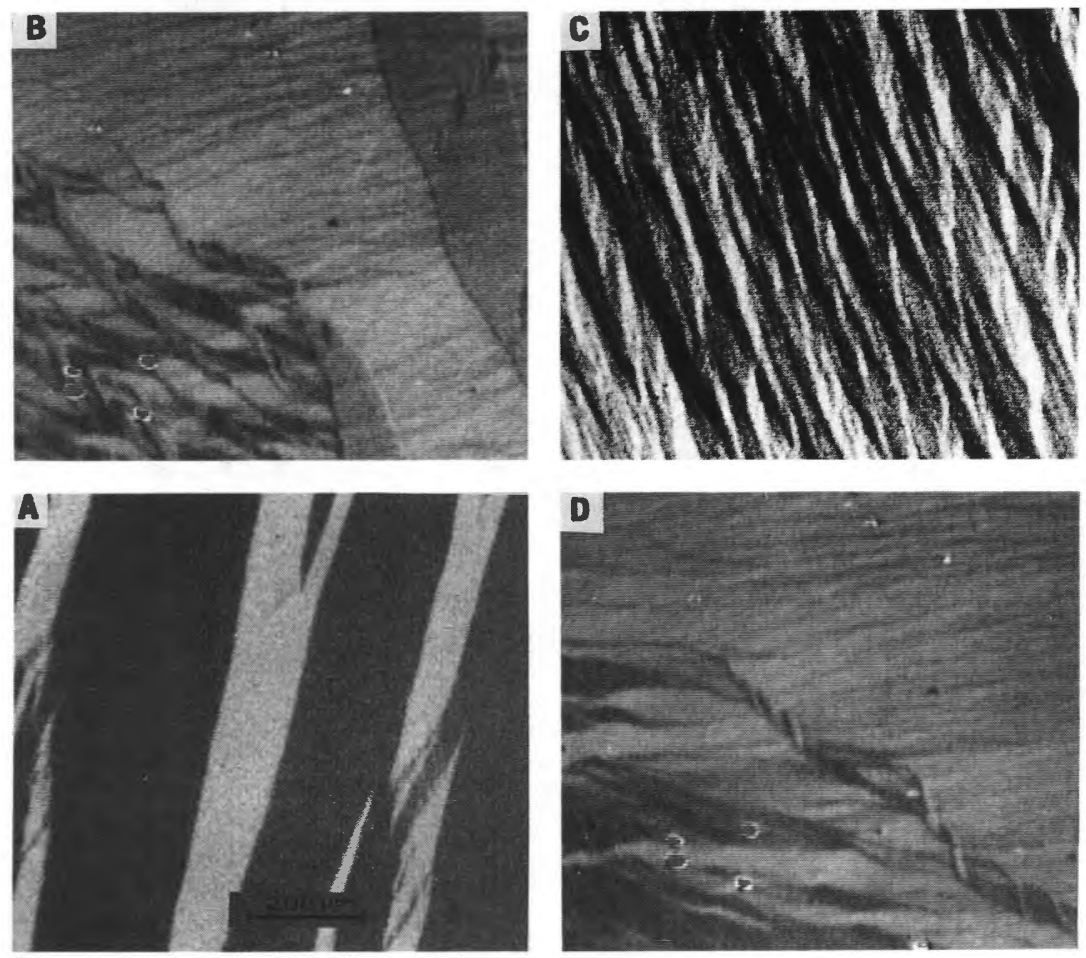

Fig. 1 : Four different remanent domain structures of Fe sample. ( All photographs have the same magnification )

applied field to a bubble structure.Furthermore, in Fig, 2B there are two big domains one black and one white. Inside of the white one there is possible to be notice the circular black domains with different diameters. Some of them seems to have the bubble dimensions. There is also interesting to point out the differences between Fig. 2A and 2B which represented the two remanent patterns before and over composition with compensation temperature near room temperature. ${ }^{12}$ In general the domain structure depends strongly on the direction of the uniaxial anisotropy and the magnitude of magnetization which is influenced by the compensation temperature. ${ }^{13}$ This statement is also confirmed by our domain observations, Fig2B2D, where the domain dimensions are gradually decreasing with increasing the Tb content far away to the composition with compensation temperature near room temperature. One of the main conclusion results from the domain observation is that samples with $0 \leq x \leq 0.23$ show in plane anisotropy, while the sample with $0.26 \leq x \leq 0.37$ show uniaxial anisotropy with the easy axis perpendicular to the film plane.A typical example of a local hysteresis loop obtained by using a He-Ne laser in the longitudinal Kerr effect for the Fe polycrystalline sample is shown in Fig. 3. Similar curves have been obtained for the sample with $0 \leq x \leq 0.23$, proving that easy axis is lying in the plane of samples. For all the samples additional $\mathrm{M}-\mathrm{H}$ loop measurements, using a VSM with the magnetic field applied perpendicular and parallel to the film surfaces were carried out . Fig. 4 shows the dependence of coercivity (Hc) versus saturation magnetization (Ms) .The sample with $x=0$ has the lowest value of $\mathrm{Hc}$ and the 

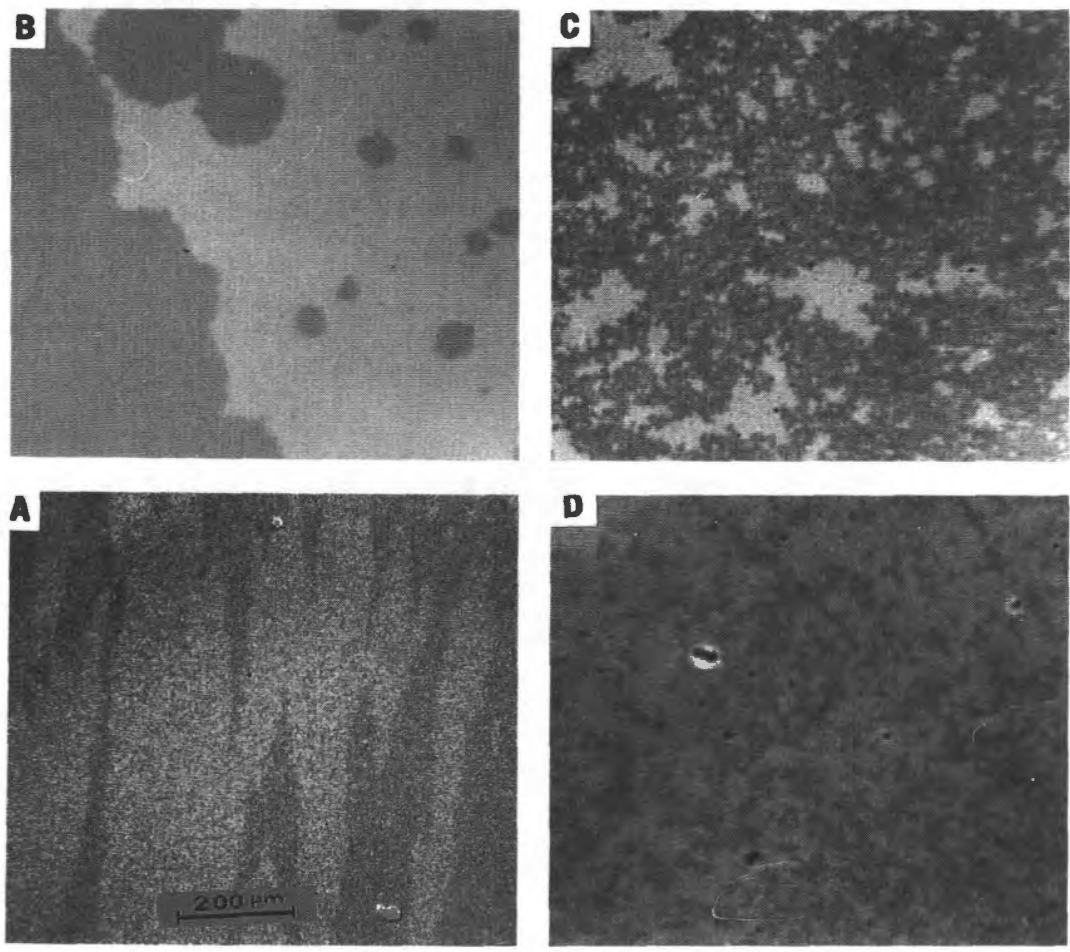

Fig. 2 : The remanent domain structures for the samples $x=0.23(A), 0.26(B), 0.34(C)$, and 0.37 (D). ( All photographs have the same magnification )

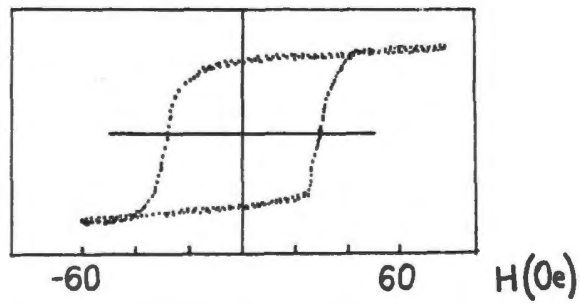

Fig. 3 : Local hysteresis loop for the sample with $x=0$

maximum value of Ms. For the samples with $0.27 \leq x \leq 0.34$ the $\mathrm{Hc}$ always has value over $1.26 \mathrm{kOe}$ with the maximum of $4.27 \mathrm{kOe}$.The quite exponential decrease of coercivity with increasing the saturation magnetization in the condition of $\mathrm{Tb}$ content variation has also reported by Higashi. ${ }^{4}$ In order to check if our Tb-Fe films with uniaxial anisotropy are suitable for obtaining high remanence magnetization required for magnetic printing we show Fig. 5 . It is evident from this that samples with $0.26 \leq x \leq 0.34$ have a very high remanence 


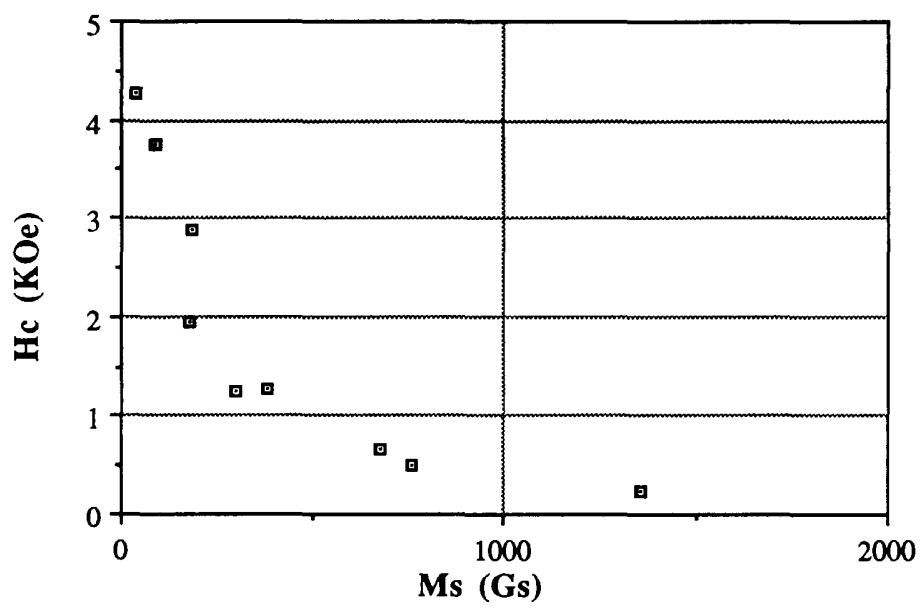

Fig. 4 : The dependence of coercivity ( $\mathrm{Hc}$ ) versus saturation magnetization ( $\mathrm{Ms}$ ) for $\mathrm{Tb}$ Fe serie samples.

magnetization with $\mathrm{Mr} / \mathrm{Ms}$ between $84 \%$ and $100 \%$. It is also possible in this Fig. to make the distinguish between samples with in- plane and uniaxial anisotropy and to confirm the domain structure observations .

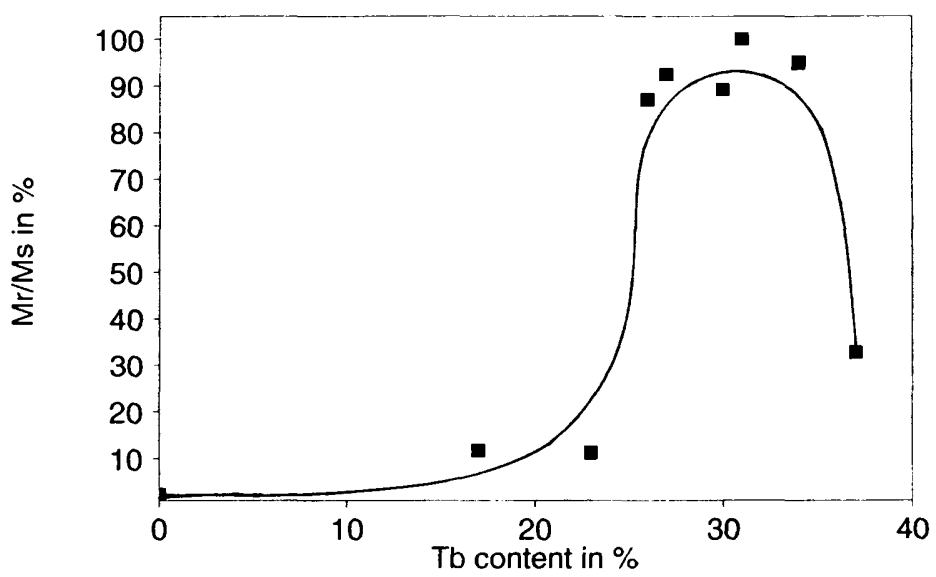

Fig. 5 : The dependence of $\mathrm{Mr} / \mathrm{Ms}$ ratio versus $\mathrm{Tb}$ content for $\mathrm{Tb}-\mathrm{Fe}$ serie samples. 


\section{CONCLUSIONS}

- Demagnetized $\mathrm{Fe}_{\mathrm{X}} \mathrm{Tb} \mathrm{l}_{1-\mathrm{X}}$ films show strongly different domain patterns below and above composition with $\mathrm{x}=0.23$.

- The magnetic domain observation and additional magnetic measurements show that samples with $0 \leq x \leq 0.23$ exhibit in- plane anisotropy, while samples with $0.26 \leq x \leq 0.37$ possess uniaxial anisotropy with easy-axis oriented perpendicular to the film plane.

- High magnetic remanence is found for samples with $0.27 \leq x \leq 0.34$.

\section{ACKNOWLEDGMENTS}

We thank Prof. A. Hubert for using the magnetic domain observation equipment and $\mathrm{Mr}$. M. Haidl for his assistance in VSM measurements. This work was supported by the National Science Foundation, the Welch Foundation, the Texas Higher Education Coordinating Board and the Alexander von Humboldt Foundation.

\section{REFERENCES}

1. P. Hansen and H. Heitmann , IEEE Trans. Magn. MAG - 25, 4390 (1989).

2. N. Imamura, S. Tanaka, F. Tanaka and Y. Nagao, IEEE Trans. Magn. MAG-21,1607 (1985)

3. M. Komori, T. Numata, K. Tsutsumi, S. Inokuchi and Y. Sakurai, IEEE Trans. Magn MAG-20, (1984).

4. J. Higashi, T. Numata, S. Inokuchi and Y. Sakuray, Japan J. Appl. Phys.,26,1262 (1987).

5. J. J. Eltgen and J. G. Maguenet, IEEE Trans. Magn. MAG - 16, 961 (1980).

6. C. J. Lin, J. C. Suit and R. H. Geiss, J . Appl. Phys. 63, 3835 (1988).

7. Y. Takeno, M Suwabe, T. Sakurai and K. Goto, Japan J. Appl. Phys. 25, L 657, (1986).

8. V. Florescu, Zaki AL - Tamimi, M. D. Serbanescu, R. Manaila and W. A. Grant, IEEE Trans. Magn. MAG -24, 1734 (1988).

9. V. Florescu, M. D. Serbanescu, R. Manaila, W. A. Grant, J. Magn. Mag. Mat., 92, 137 (1990).

10. F. Schmidt, W. Rave and A. Hubert, IEEE Trans . Magn. MAG - 21, 1596 ( 1985).

11. B. D. Cullity, Introduction to Magnetic Materials (Addison - Wesley Publishing Comp. Inc., 1972 ) p. 436.

12. P. Hansen, C. Clausen, G. Much, M. Rosenkranz and K. Witter, J. Appl. Phys. 66, 756 (1989).

13. P. Hansen, J. Appl. Phys. 63, 2364 (1988). 